\title{
New proportionate affine projection sign algorithms
}

\author{
Felix Albu \\ Faculty of Electrical Engineering \\ Valahia University of Targoviste \\ Targoviste, Romania \\ felix.albu@valahia.ro
}

\author{
H. K. Kwan \\ Department of Electrical and Computer Engineering \\ University of Windsor \\ Windsor, Canada \\ kwan1@uwindsor.ca
}

\begin{abstract}
In this paper, two proportionate affine projection sign algorithms are introduced. The performance of the proposed algorithms is compared with that of other proportionate affine algorithms under impulsive interference environment of a network echo cancellation system. It is shown that one of the proposed algorithms, termed memory improved proportionate affine projection sign algorithm (MIP-APSA), is the most robust to impulsive interferences and colored inputs. It is proved that MIP-APSA is a good candidate for network echo cancellation, because of its low complexity, good convergence speed and tracking abilities for echo paths with different sparseness measures, and projection orders.
\end{abstract}

\section{INTRODUCTION}

It is known that, in applications such as network echo cancellation, the echo paths are typically long and sparse. Adaptive filters are used to identify the echo paths and many adaptive filtering algorith $m s$ have been proposed (e.g. [1] and the references therein). The proportionate normalized least mean square algorithm (PNLMS) [2] and its improved IPNLMS version [3] exploits the sparseness of the echo paths. An improved algorithm, called the $\mu$-law PNLMS, used the logarithm of the coefficient magnitudes instead of coefficient magnitudes [4]. It is also known that the families of affine projection algorithms (APA) [5-7], and proportionate affine projection algorithms (PAPA) [8-15] have superior performances over the NLMS type of algorithms. Examples of PAPA are the Improved Proportionate APA (IPAPA) [9], the Memory Improved Proportionate APA (MIPAPA) [10], and the efficient Approximated MIPAPA (AMIPAPA) [11] etc. The MIPAPA and the AMIPAPA use previously computed proportionate coefficients in order to reduce the overall complexity of PAPA. The same idea was used in [12] in order to derive the $\mu$-law version of MIPAPA, called the $\mu$-law MIPAPA (MMIPAPA). The MMIPAPA showed further performance improvements over APA/IPAPA/MIPAPA [12], but at the cost of added logarithmic operations. Other proportionate algorithms with reduced complexity but with lower convergence performance were recently proposed in [13-15].

This work was supported by a grant of the Romanian National Authority for Scientific Research, CNCS - UEFISCDI, project number PN-II-ID-PCE2011-3-0097
Unfortunately, the APA and PAPA family of algorithms are not very robust to impulsive noise interference [16-20]. Robust affine projection sign algorithms (APSA) against outliers have been proposed in [16] and [17]. Recently, the real-coefficient improved proportionate affine projection sign algorithm (RIP-APSA) was presented and proved to be robust to such outliers [18-19]. The RIP-APSA was obtained from an $l_{1}$-norm optimization. In [19] The RIP-APSA's superiority over APA, APSA and PAPA's in terms of robustness and fast convergence for both sparse and dispersive echo paths in impulsive environments was proved by many simulation results. In the next section, two new proportionate affine sign algorithms are presented: The Memory Improved Proportionate Affine Projection Sign Algorithm (MIP-APSA) and the $\mu$-law Memory Improved Proportionate Affine Projection Sign Algorithm versions, termed MMIP-APSA. Simulation results and conclusions are included in this paper.

\section{ALGORITHMS DESIGN}

The proposed algorithms are derived starting from the RIP-APSA [18]. The adaptive filter that models the true $L$ length echo path, $\mathbf{h}, \quad$ is defined by $\mathbf{w}(k)=\left[w_{0}(k), w_{1}(k), \ldots, w_{L-1}(k)\right]^{T}$, where superscript $T$ denotes transposition, and $k$ is the time index. We denote $x(k), z(k)$ and $v(k)$ as the far-end, the near-end and background noise signals, respectively. The desired signal is $y(k)=\mathbf{x}^{T}(k) \mathbf{h}+z(k)+v(k) \quad$ where the vector $\mathbf{x}(k)=[x(k), \ldots, x(k-L+1)]^{T}$ collects the far-end signal. The output of the adaptive filter is $\hat{y}(k)=\mathbf{x}^{T}(k) \mathbf{w}(k)$, and the error vector is given as

$$
\mathbf{e}(k)=\mathbf{y}(k)-\mathbf{X}^{T}(k) \mathbf{w}(k),
$$

where $\mathbf{y}(k)=[y(k), y(k-1), \ldots, y(k-M+1)]^{T}$ is a $M \times 1$ vector, $M$ is the projection order, and 
$\mathbf{X}(k)=[\mathbf{x}(k), \mathbf{x}(k-1), \ldots, \mathbf{x}(k-M+1)]$ is the input signal matrix. It is known that sign based adaptive algorithms can suppress impulsive noise components [16]-[19].

For the RIP-APSA [18], the filter coefficients were adapted proportionately by pre-multiplying the update vector with the proportionate matrix $\mathbf{G}(k)=\operatorname{diag}\left\{g_{0}(k), \ldots, g_{L-1}(k)\right\}$ which contains the proportionate factors, $g_{l}(k)$ defined as:

$$
g_{l}(k)=\frac{1-\alpha}{2 L}+(1+\alpha) \frac{\left|w_{l}(k)\right|}{2 \sum_{i=0}^{L-1}\left|w_{i}(k)\right|+\varepsilon}, l=1 . . L .
$$

where $-1<\alpha<1$ and $\varepsilon$ is a small positive constant to avoid division by zero [18].

The RIP-APSA [18] co mputes:

$$
\mathbf{x}_{g s}(k)=\mathbf{G}(k) \mathbf{X}(k) \operatorname{sgn}(\mathbf{e}(k)),
$$

where $\operatorname{sgn}(\cdot)$ is the signum function.

The weight updating equation for the RIP-APSA is

$$
\mathbf{w}(k+1)=\mathbf{w}(k)+\frac{\mu \mathbf{x}_{g s}(k)}{\sqrt{\delta+\mathbf{x}_{g s}^{T}(k) \mathbf{x}_{g s}(k)}},
$$

where $\mu$ is the step size and $\delta$ is another small constant to avoid division by zero [18].

If we note $\mathbf{P}(k)=\mathbf{G}(k) \mathbf{X}(k)$ we notice that

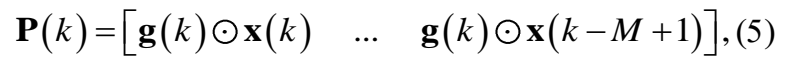

where $\mathbf{g}(k)=\left[g_{0}(k), \ldots, g_{L-1}(k)\right]^{T}$ and the operator $\odot$ denotes the Hadamard product. As in [10], the "proportionate history" from the last $M$ moments of time is taken into account and $\mathbf{P}(k)$ is approximated by $\mathbf{P}^{\prime}(k)$ defined as

$$
\mathbf{P}^{\prime}(k)=[\mathbf{g}(k) \odot \mathbf{x}(k) \quad \ldots \quad \mathbf{g}(k-M+1) \odot \mathbf{x}(k-M+1)] .
$$

The $L \times M \quad \mathbf{P}^{\prime}(k)$ matrix can be computed recursively as $\mathbf{P}^{\prime}(k)=\left[\mathbf{g}(k) \odot \mathbf{x}(k) \quad \mathbf{P}_{-1}^{\prime}(k-1)\right]$, where the matrix

$$
\mathbf{P}_{-1}^{\prime}(k)=[\mathbf{g}(k-1) \odot \mathbf{x}(k-1) \ldots \mathbf{g}(k-M+1) \odot \mathbf{x}(k-M+1)],
$$

contains the first $M-1$ columns of $\mathbf{P}^{\prime}(k-1)$ [11].
TABLE I. NUMBEROFMULTIPLICATIONS OF INVESTIGATED ALGORITHMS

\begin{tabular}{|c|c|c|c|}
\hline MIP-APSA & RIP-APSA & MMIP-APSA & MMIPAPA \\
\hline$(M+4) \times L$ & $(M+4) \times L$ & $(M+5) \times L$ & $(4 M+2) \times L+M^{2}+M$ \\
& & & \\
\hline
\end{tabular}

An approximate $L \times 1$ vector $\mathbf{x}_{g^{\prime} s}(k)$ is then computed as follows:

$$
\mathbf{x}_{g^{\prime} s}(k)=\mathbf{P}^{\prime}(k) \operatorname{sgn}(\mathbf{e}(k)) .
$$

Finally, the weight updating equation for the MIP-APSA is

$$
\mathbf{w}(k+1)=\mathbf{w}(k)+\frac{\mu \mathbf{x}_{g^{\prime} s}(k)}{\sqrt{\delta+\mathbf{x}_{g^{\prime} s}^{T}(k) \mathbf{x}_{g^{\prime} s}(k)}} .
$$

The computation of $\mathbf{x}_{g^{\prime} s}(k)$ requires the same number of multiplications and additions as $\mathbf{x}_{g s}(k)$, therefore, the numerical comple xity of the MIP-APSA is the same with that of the RIP-APSA.

The logarithmic proportionate updating scheme ([4] and [12]) can be easily incorporated in the MIP-APSA by modifying the proportionate coefficients of (2) as in (10)-(11):

$$
g_{l}(k)=\frac{1-\alpha}{2 L}+(1+\alpha) \frac{\left|\mathrm{F}\left(w_{l}(k)\right)\right|}{2 \sum_{i=0}^{L-1}\left|\mathrm{~F}\left(w_{i}(k)\right)\right|+\varepsilon}, l=1 . . L,(10)
$$

$$
\mathrm{F}\left(w_{l}(k)\right)=\ln \left(1+\mu_{\log }\left|w_{l}(k)\right|\right) .
$$

As in [12], a value of $\mu_{\log }=1000$ is used. Therefore, a new sign algorithm termed the $\mu$-law MIP-APSA (MMIP-APSA) is obtained.

The MMIP-APSA requires additional $L$ logarithmic functions and $L$ additions per iteration in comparison with the MIP-APSA. The overall complexity of the investigated algorithms is shown in Table 1. By far the most complex algorithm is the MMIPAPA. Depending on $L$ and $M$ values, the MMIPAPA can have almost three times more multiplications than the PAPSA algorithms.

\section{SimULATION RESULTS}

The MMIPAPA [12], the RIP-APSA [19], the MIP-APSA, and the MMIP-APSA were simulated in impulsive interference environment and their speed of convergence and tracking abilities were compared. We used $\delta=\varepsilon=0.01, \alpha=0$ in the simulations of sign based adaptive algorith ms. 

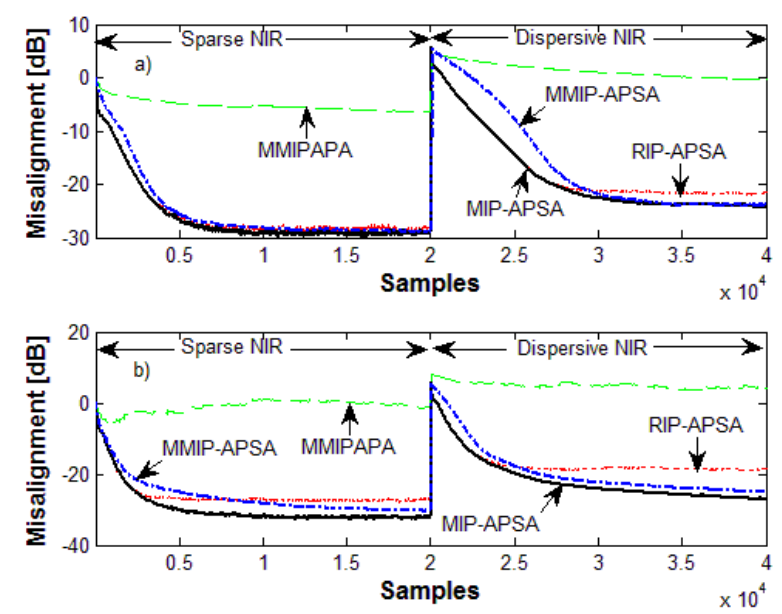

Figure 1. Misalignment of RIP-APSA, MIP-APSA, MMIP-APSA and MMIPAPA with colored input signal, $\operatorname{Pr}=0.001$ and $\mu=0.01$. a) $M=2$; b) $M=8$.

The input signal was an $\mathrm{AR}(1)$ signal with a pole at 0.8 and a white noise was added to the near-end in order to have a signal-to-noise ratio $\mathrm{SNR}=35 \mathrm{~dB}$. The near end signal $z(k)$ has a signal-to-interference ratio $\mathrm{SIR}=-10 \mathrm{~dB}$ and is modeled by a Bernoulli-Gaussian (BG) signal [16]. The BG distribution was generated as in [19] being a product of a Bernoulli process with the parameter $\operatorname{Pr}$ and a Gaussian process, keeping its average power constant.

The simulations were performed using two network impulse responses (NIR) with $L=512$ coefficients with different sparseness measures defined by $\xi=(L / L-\sqrt{L}) \cdot\left(1-\|\mathbf{h}\|_{1} / \sqrt{L}\|\mathbf{h}\|_{2}\right)$, where $0 \leq \xi \leq 1 \quad[21]$. The sparse echo path has $\xi=0.850$ and the dispersive echo path has $\xi=0.556$. The performance of the algorithms was measured by the normalized misalignment defined by $\eta(k)=20 \cdot \log _{10}\left(\|\mathbf{h}-\mathbf{w}(k)\|_{2} /\|\mathbf{h}\|_{2}\right)$ averaged over 10 experiments.

The convergence and tracking performance of the APSA, the RIP-APSA, the MMIP-APSA and the MIP-APSA using $\mu=0.01$ for all the algorithms, in the $\mathrm{BG}$ interference environment with $\operatorname{Pr}=0.001$ is shown in Fig. 1. The echo path changed from the sparse NIR to dispersive NIR after 20000 samples. Two projection orders were used and, as expected, the convergence speed of both algorithms increases with $M$. It can be seen that the MIP-APSA obtains the lowest steadystate misalignment and has similar tracking abilities with the RIP-APSA. Fig. 1 shows the clear superiority of the MIPAPSA over the MMIP-APSA and the MMIPAPA in impulsive interference environments in both convergence speed and tracking abilities. It was mentioned in [19] that the step size of the RIP-APSA is recommended to be smaller than 0.1 for practical NEC applications.
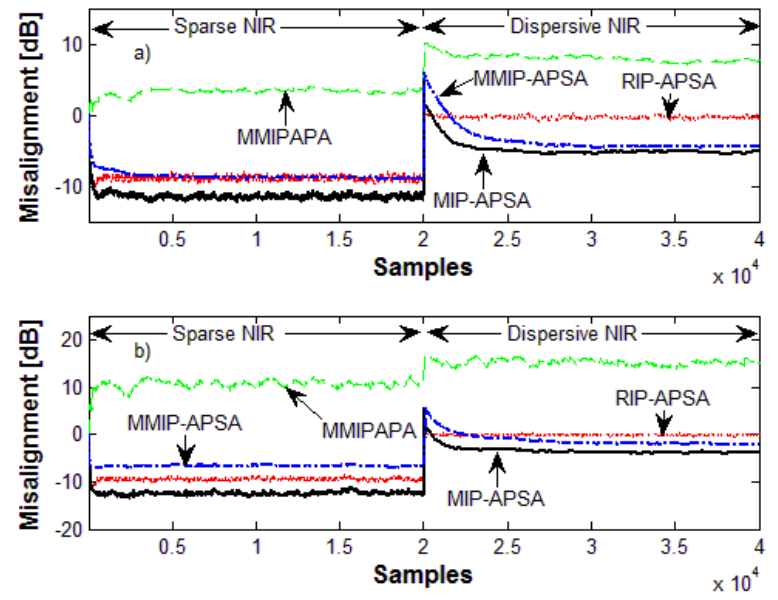

Figure 2. Misalignment of RIP-APSA, MIP-APSA, MMIP-APSA and MMIPAPA with colored input signal, $\operatorname{Pr}=0.01$ and $\mu=0.1$. a) $M=2$; b) $M=8$.

In Fig. 2 the influence of a stronger BG interference of $\operatorname{Pr}=0.01$ on the APSA, the RIP-APSA, the MMIP-APSA and the MIP-APSA convergence is examined for $\mu=0.1$ and two projection orders $(M=2$ and $M=8)$. It can be seen that the MIP-APSA has the best steady-state performance among the four algorithms. Also, it can be noticed from Figs. 1 and 2 that a larger step size lead to a faster convergence of the MIPAPSA, but to a higher steady-state misalignment too. The same conclusions were obtained for different in itializations of the weight vector, filter lenghts and the performance improvement can be even higher than $10 \mathrm{~dB}$ for high projection orders. It can be seen from Figs. 1 and 2 that the tracking performance of the MIP-APSA is close to that of the RIP-APSA. Therefore, the MIP-APSA has the best performance in case of changing echo path sparseness or BG interference levels. Fig. 2 also confirms the instability of the MMIPAPA in impulsive environments.

The performance of the four algorithms for speech signals with a double talk situation is also investigated. A Geigel detector was used for all algorithms in order to detect the double talk situation assuming $6 \mathrm{~dB}$ attenuation and the hangover time $T_{\text {hold }}=240$ samples [22]. The double talk happened between the samples 15000 and 25000 and the average far-end to double talk ratio is $\mathrm{SIR}=-8 \mathrm{~dB}$ and $\mathrm{SNR}=35 \mathrm{~dB}$.

Fig. 3 confirms the previous results and suggests that a more robust DTD to impulsive noise should be used. For the investigated double talk case and chosen parameters, the MIP-APSA is about $40 \%$ less complex than the MMIPAPA in terms of multip lications. 


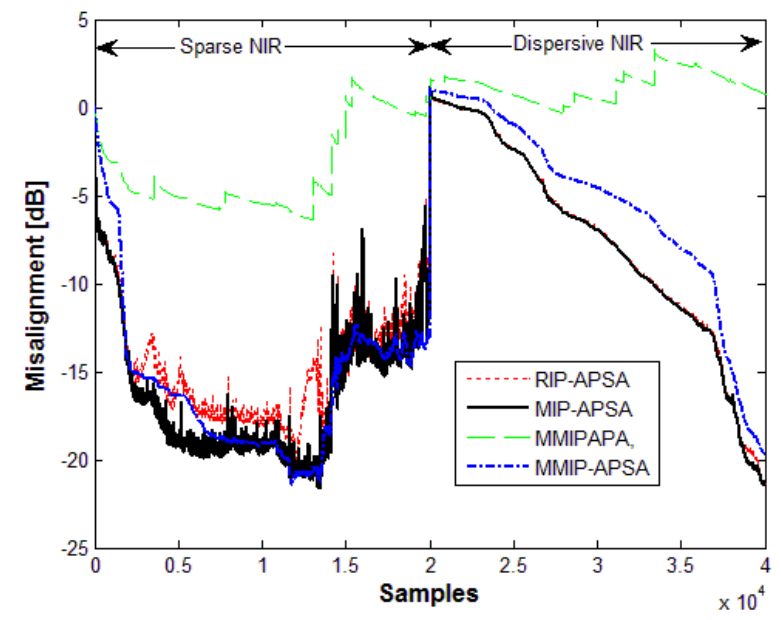

Figure 3. Misalignment of RIP-APSA, MIP-APSA, MMIP-APSA and MMIPAPA using the Geigel DTD, with speech input signal, $\operatorname{Pr}=0.001$,

$$
M=2 \text { and } \mu=0.005 \text {. }
$$

Our simulations have indicated that the values of $\alpha=0$ or $\alpha=0.5$ lead to the best performance of MIP-APSA or MMIP-APSA. The performance of the proposed algorithms is slightly reduced for small projection orders in case of using very small $\delta$ or $\varepsilon$ values. Also, other simulations (not shown here due to the lack of space) have imdicated that the speed of MMIP-APSA convergence can be slightly increased by using a smaller $\mu_{\log }$ value, but the steady-state misalignment could increase a bit too. Our future work will be focused on improving the proposed algorithms and investigate their use in image processing [23] or hearing aids [24] applications.

\section{CONCLUSIONS}

In this paper, two proportionate-type affine projection sign algorithms namely, the MIP-APSA and the MMIP-APSA are proposed. They use a recursive procedure and the previous proportionate factors. It has been proved that the MIP-APSA offers the best performance/complexity ratio among the all considered algorithms for echo paths with different sparseness measures, projection orders and BG interference levels.

\section{REFERENCES}

[1] E. Haensler and G. Schmidt, Eds., Topics in Acoustic Echo and Noise Control Berlin, Germany: Springer-Verlag, 2006.

[2] D. L. Duttweiler, "Proportionate normalized least-mean-squares adaptation in echo cancellers", IEEE Trans. Speech Audio Process., vol. 8, no. 5, pp. 508-518, September 2000.

[3] J. Benesty and S. L. Gay, "An improved pnlms algorithm", in Proc. of ICASSP 2002, 2002, vol. 2, May 2002, pp. $1881-1884$.

[4] H. Deng and M. Doroslovacki,"Proportionate adaptive algorithms for net work echo cancellation", IEEE Trans. Signal Process., vol. 54, no. 5, pp. 1794-1803, May 2006.

[5] K. Ozeki and T. Umeda, "An adaptive filtering algorithm using an orthogonal projection to an affine subspace and its properties", Electron. Commun. Jpn., vol. 67-A, no. 5, pp. 19-27, 1984.
[6] A. Gonzalez, M. Ferrer, F. Albu, and M. de Diego, "Affine projection algorithms: evolution to smart and fast multichannel algorithms and applications", in Proc. of Eusipco 2012, pp. 1965-1969.

[7] F. Albu and H.K. Kwan, "Fast block exact Gauss-Seidel pseudo affine project ion algorithm", Electronics Letters, vol. 40, issue 22, pp. 1451 1453, Oct. 2004.

[8] T. Gansler, J. Benesty, S.L. Gay, and M. Sondhi,"A robust proportionate affine projection algorithm for network echo cancellation," in Proc. of ICASSP 2000, 2000, pp. 793-796.

[9] O. Hoshuyama, R. A. Goubran, and A. Sugiyama, "A generalized proportionate variable step-size algorithm for fast changing acoustic environments", in Proc. of ICASSP 2004, 2004, vol. 4, pp. 161-164.

[10] C. Paleologu, S. Ciochina, and J. Benesty, "An efficient proportionate affine projection algorithm for echo cancellation", IEEE Signal Processing Letters, vol. 17, no. 2, pp. 165-168, Feb. 2010.

[11] F. Albu, C. Paleologu, J. Benesty, and S. Ciochina, "A low complexity proportionate affine projection algorithm for echo cancellation", in Proc. EUSIPCO 2010, 2010, pp. 6-10.

[12] J. Yang and G.E. Sobelman, "Efficient $\mu$-law improved proportionate affine projection algorithm for echo cancellation", Electronics Lett., vol. 47, issue 2, pp. 73-74, January 2010.

[13] F. Albu, "Simplified proportionate affine projection algorithms", in Proc. of IWSSIP 2012, 2012, pp. 382-385.

[14] F. Albu, "A proportionate affine projection algorithm using fast recursive filtering and dichotomous coordinate descent iterations", in Proc. of SPAMEC 2011, 2011, pp. 93-96.

[15] F. Albu, "New proportionate affine projection algorithm", in Proc. of Inter-Noise and Noise-Con Congress and Conference, 2012, vol. 11, pp. 40-46.

[16] T. Shao, Y. R. Zheng, and J. Benesty, "An affine projection sign algorithm robust against impulsive interferences", IEEE Signal Processing Lett., vol. 17, no. 4, pp. 327-330, Apr. 2010.

[17] S. J. Ban, S. W. Kim, "Pseudo affine projection sign algorithm for robust system identification", IET Electronics Lett., vol. 46, no. 12 , pp. 865-866, June 2010.

[18] Z. Yang, Y. R. Zheng, and S. L. Grant, "Proportionate affine projection sign algorithms for net work echo cancellation", IEEE Transactions on Audio, Speech, and Language Processing, vol. 19, no. 8, pp. 2273 2284, November 2011.

[19] Z. Yang, Y. R. Zheng, and S. L. Grant, "Proportionate affine projection sign algorithms for sparse system identification in impulsive interference", in Proc. of ICASSP 2011, 2011, pp. 4068-4071.

[20] F. Albu and H. K. Kwan, "Memory improved affine projection sign algorithm", IET Electronics Letters, vol. 48, Issue 22, pp.1279-1281, 27 September 2012.

[21] P. O. Hoyer, "Non-negative matrix factorization with sparseness constraints", J. Mach. Learn. Res., 2004, vol. 5, pp. 1457-1469.

[22] T. Gansler, S. Gay, M. Sondhi, and J. Benesty, "Double-talk robust fast converging algorithms for network echo cancellation," IEEE Trans. Speech, Audio Processing, vol. 8, no. 6, pp. 656 -663, November 2000.

[23] F. Albu, C. Florea, A. Drimbarean, A. Zamfir, "Adaptive recovery of motion blur point spread function from differently exposed images", Proc. of IS\&T/SPIE EI 2010, 2010, 7537-25.

[24] M. Rotaru, F. Albu, H. Coanda, "A Variable Step Size Modified Decorrelated NLMS Algorithm for Adaptive Feedback Cancellation in Hearing Aids," in Proc. of ISET C 2012, 2012, pp. 263-266.

The codes for the proposed algorithms can be obtained from http://falbu.50webs.com/List of publications aec.htm

The reference for the paper is:

F. Albu, H.K.Kwan, "New proportionate affine projection sign algorithms", in Proc. of ISCAS 2013, Beijing, China, pp. $1789-1793$ 